\title{
Auto detecting deliveries in elite cricket fast bowlers using microsensors and machine learning
}

\author{
${ }^{1}$ Hannah K Jowitt, ${ }^{2}$ Jérôme Durussel, ${ }^{1}$ Raphael Brandon and ${ }^{3}$ Mark A King
}

${ }^{1}$ England and Wales Cricket Board, ${ }^{2}$ Catapult Sports, ${ }^{3}$ School of Sport, Exercise and Health Sciences, Loughborough University, Leicestershire, LE11 3TU, UK

\begin{abstract}
Cricket fast bowlers are at a high risk of injury occurrence, and this has previously been shown to be correlated to bowling workloads. This study aimed to develop and test an algorithm that can automatically, reliably and accurately detect bowling deliveries. Inertial sensor data from a Catapult OptimEye S5 wearable device was collected from both national and international level fast bowlers $(n=35)$ in both training and matches, at various intensities. A machine-learning based approach was used to develop the algorithm. Outputs were compared with over 20,000 manually recorded events. A high Matthews correlation coefficient $(r=0.945)$ showed very good agreement between the automatically detected bowling deliveries and manually recorded ones. The algorithm was found to be both sensitive and specific in training $(96.3 \%, 98.3 \%)$ and matches $(99.6 \%, 96.9 \%)$, respectively. Rare falsely classified events were typically warm-up deliveries or throws preceded by a run. Inertial sensors data processed by a machine-learning based algorithm provide a valid tool to automatically detect bowling events, whilst also providing the opportunity to look at performance metrics associated with fast bowling. This offers the possibility to better monitor bowling workloads across a range of intensities to mitigate injury risk potential and maximise performance.
\end{abstract}

Keywords: algorithm; GPS; workload

\section{INTRODUCTION}

Cricket is a global sport in which two teams of eleven players compete against each other, with each player usually specialising in batting and/or bowling. Bowlers are classified by their intention: fast or spin. Fast bowlers aim to deliver the ball as quickly as possible in order to minimise the time the batter has to select and execute the appropriate shot. The fast bowling action consists of several phases; a run-up, a predelivery stride in which the bowler bounds into the air, the bowling stride which consists of both back foot and front foot contact where the bowler lands with a braced front knee, ball release where the arm is brought in a circular motion above the head and released with a straight arm, and the follow through (Ferdinands, Marshall \& Kersting, 2010, Worthington, King, \& Ranson, 2013). Internationally there are three different formats; multiday, 50 over and Twenty 20 cricket. The differing workload demands of these formats have previously been researched (Petersen, Pyne, Portus, Karppinen \& Dawson, 2009; Petersen, Pyne, Dawson, Portus \& Kellett, 2010). High workloads in cricket have been linked with a higher risk of injury (Dennis, Farhart, Goumas \& Orchard, 2003; Orchard, James, Portus, Kountouris \& Dennis, 2009). Fast bowlers have been found to have higher workloads than other positions within the team and so are at greater risk of injury (Petersen, Portus \& Dawson, 2009; Petersen et al., 2010; Ranson, Hurley, Rugless, Mansingh \& Cole, 2013). This is due to the forces exerted upon the body during a delivery, particularly the lower limbs and lower back (Orchard et al., 2015; Ranson, Burnett, King, Patel \& O'Sullivan, 2008).

Previous research has found that fast bowlers have an increased risk of injury if they are not fully prepared i.e. bowl too little, or if they have too high a bowling workload ie. bowl too much, suggesting there is an optimum threshold for fast bowlers to minimise injury potential (Dennis et al., 2003; Orchard et al., 2009). More recent 
research looking into acute ( 7 day) and chronic (28 day) workloads has found that the rate of bowling workload change is a better indicator of injury risk than the previous absolute values used (Hulin, Gabbett, Blanch, Chapman, Bailey \& Orchard, 2014; Warren, William, McCaig \& Trewartha, 2018). To evaluate the risk, self-reporting, by either the bowler or coach, has typically been used to monitor bowling workloads. This is a simple method, but its effectiveness is limited due to accuracy issues caused by human error, particularly with training deliveries. Another potential limitation with selfreporting is that it assumes all deliveries are bowled with the same intensity, whereas training deliveries are often bowled at a lower intensity than match deliveries (Petersen, Pyne, Dawson, Kellett \& Portus, 2011). By recording all bowling deliveries at the same intensity, a fast bowler's workload could be over or underestimated, increasing the risk of injury. The limitation on recording workload intensity also impacts return to play protocols. With the differing demands of the three formats of the game, it is important that fast bowlers are prepared for the workload required of them at match intensity. Hence, it is important to find an improved method for the monitoring of fast bowling workloads, that also looks to consider the intensity of the deliveries being bowled (Hulin et al., 2014; Warren et al., 2018).

Recent years have seen an increase in the use of Global Positioning System (GPS) units and inertial sensors (accelerometers and gyroscopes) within sport. In cricket GPS units have been used to track the movements of players across all three formats of the game to develop an understanding of the difference in workloads and intensities between the formats (Petersen et al., 2010).

Further developments have seen algorithms used to detect specific events within a sport to aid in the monitoring of workloads including rugby league collisions and tackles in Australian football (Gastin, McLean, Spittle \& Breed, 2013; Hulin, Gabbett, Johnston \& Jenkins, 2017). In 2015, McNamara, Gabbett, Chapman, Naughton \& Farhart, investigated the ability of a linear algorithm to automatically detect fast bowling deliveries in cricket, during both training and competition. The algorithm was found to have a sensitivity of $99.0 \%$ and $99.5 \%$, and specificity was $98.1 \%$ and $74.0 \%$, in training and competition respectively. This algorithm was deemed to be an effective method of monitoring bowling workloads. However, the study did have some limitations. One potential reason for the high number of false positives detected in competition was that the data was collected primarily in a training environment, manufacturing non-bowling events. The same data set was also used to train and determine the performance of the algorithm which increases the risk of over fitting and can inflate performance scores (Chicco, 2017).

Pfeifer \& Hohmann (2012) discussed the limitations of the simple linear model algorithms, such as the one used by McNamara et al. (2015), in detecting human movements and propose that more complex, non-linear models are needed. Machine learning algorithms have been utilised in several studies, using data from sporting movements, to predict and classify sporting events, and also to analyse and model sporting performance (Kelly, Coughlan, Green \& Caulfield, 2012). Whiteside, Cant, Connolly \& Reid (2017) developed an algorithm, using a support vector machine model (SVM), to automatically detect and discriminate between different tennis strokes. It was found that combining machine learning and accelerometer data provided a valid and automated solution for monitoring tennis hitting loads. More recently McGrath, Neville, Stewart \& Cronin (2019) have used several different machine learning models including random forest and SVM to detect cricket fast bowling deliveries in a training environment. The results showed that all models had high specificity and sensitivity ranging from 93 to $100 \%$ and no model was consistently better than the others. However, despite making advances, some of the methodological limitations observed 
by McNamara et al., (2015) remain. Specifically, McGrath et al. (2019) manufactured non-bowling events in a training context only. Other recent studies (McNamara et al., 2015; Murray, Black, Whiteley, Gahan, Cole, Utting \& Gabbett, 2016) have identified a significant decrease in accuracy of detection of events during competition so it's likely the accuracy levels observed by McGrath et al. (2019) would decrease when used in competition. Whilst McGrath et al. (2019) did not use the same data sets for training and validating the algorithm, the training and validation groups were not truly independent as some participants featured in both, which increases the risks of over fitting and inflation of accuracy scores (Chicco, 2017).

Machine learning algorithms have not only been utilised to predict and classify events, but also to analyse sporting performance by providing feedback information. This could be utilised within fast bowling to provide information for performance analysis purposes such as run up speed, deceleration and trunk rotation, all of which are performance metrics associated with fast bowling (Worthington et al., 2013). This could assist in the potential to begin to observe if deliveries are being bowled at different intensities, hence enabling better workload management in preparation for the demands of the game. Therefore, the main purpose of this study was to develop a sensitive and specific method of auto detection of fast bowling deliveries, in both training and match environments, using a robustly validated algorithm that utilises machine learning to monitor workload and also open the opportunity to provide performance feedback. Additionally, based on previous work in this area, this study aimed to independently validate the algorithm, with data from participants that were not used during the training and the optimisation stages of the algorithm, as well as including a large sample of data representative of both 'real' training and match environments.

\section{METHODS}

\section{Participants}

During Thirty-five professional fast bowlers (International, $n=18$, County, $n=17$ ) participated in this study, twenty-one in the development and fourteen in the validation of the algorithm. The bowlers ages ranged from 18 to 35, including professional youth fast bowlers, a combination of male $(n=30)$ and female $(n=5)$ fast bowlers were used, and also a combination of right handed $(n=31)$ and left handed $(n=4)$. At the time of the study all participants were free from injury and any other medical conditions. Loughborough University Ethics Committee approved all procedures (Approval Number SSEHS-PG42).

\section{Design}

In this cross-sectional study, video, time stamped delivery information, GPS and inertial sensor data were collected to develop and train a random forest algorithm to automatically detect fast bowling deliveries.

GPS and inertial sensors data were collected in both match and training sessions. Players wore a Catapult OptimEye S5 unit (Catapult Innovations, Melbourne, Australia) in a vest with the unit positioned on their upper back, between their shoulder blades. Triaxial accelerometers with a range of $3 \mathrm{D}+/-16 \mathrm{~g}$, gyroscopes with a range of $3 \mathrm{D}$ $2000 \mathrm{deg} / \mathrm{second}$, and magnetometers were also housed within the unit. These sampled at $100 \mathrm{~Hz}$ whilst the GPS data was sampled at $10 \mathrm{~Hz}$. Video footage was collected in matches from the live footage stream provided to team analysts. In training, video footage was collected by placing a camera (Casio Exilim EX-ZR1000) on a tripod behind the cricket nets to ensure all nets were visible in the same video frame. Time 
stamped delivery data was provided by Hawk-Eye (Hawk-Eye Innovations, UK) post International matches. This video and time stamped data allowed true and false deliveries to be manually detected.

\section{Development of the algorithm}

Using an iterative process, three main versions of the algorithm were developed and gradually optimised based on the results of each version. For version one, data was collected from four international, male right-handed bowlers during matches. Version two comprised of data from the same original four bowlers plus seven additional giving a total of eleven male international fast bowlers, both right handed $(n=9)$ and left handed $(n=2)$ during thirty-three matches. Finally, the third version comprised of data again from the same bowlers in version 2 with additional bowlers giving a total of twenty-one, both male $(n=18)$ and female $(n=3)$, and right handed $(n=18)$ and left handed $(n=3)$. This data was collected over a total of 278 sessions, from 198 matches and 81 training sessions.

A third order low-pass $5 \mathrm{~Hz}$ Butterworth filter was applied to the inertial sensors data to reduce noise. Features were then calculated from the GPS as well as the three axes of the accelerometer and the gyroscope during the long, -7 to 5.5 seconds, and short, -0.5 to 0.5 seconds, windows before and after the delivery respectively. The calculated features included minimum, maximum, median, 10th, 25th, 75th, 90th percentile and standard deviation. The random forest classifier (Breiman, 2001) was used in each version of the algorithm as the machine-learning predictive model. Random forest model previously showed high level accuracy similar to other models that were compared (McGrath et al., 2019) and was chosen mainly because of its versatility, good performance with little tuning required and robustness to overfitting (Hastie, Tibshirani and Friedman, 2009). The R package Variable Selection Using Random Forests (VSURF) (Geneur, Poggi \& Tuleau-Malot, 2015) was used to perform variable selection of the aforementioned features of the random forest model and eventually led to the 8 features presented in Table 1. The hyper-parameters of the random forest model were fine-tuned and optimised using 10 fold cross-validation to minimise over-fitting. $R$ statistical software package (version 3.5.1) was used throughout the development of the algorithm. This iterative process led to the following and final version of the algorithm.

Table 1. Eight features identified for use in algorithm.

\begin{tabular}{llr}
\hline & \multicolumn{2}{c}{ Feature Name } \\
1 & Roll_b_abs_w_max & Maximum absolute value of filtered roll rotation within the long ${ }^{*}$ window. \\
2 & Side_b_abs_sw_0.75P & $75^{\text {th }}$ percentile of absolute value of filtered side acceleration within the short ${ }^{*}$ window. \\
3 & Roll_b_abs_sw_sd & Standard deviation of filtered roll rotation within the short window. \\
4 & Side_b_abs_sw_sd & Standard deviation of filtered side acceleration within the short window. \\
5 & SmoothedVelocity_r_sw_0.25P & $25^{\text {th }}$ percentile of smoothed GPS velocity within the short window. \\
6 & Side_b_abs_w_max & Maximum absolute value of filtered side acceleration within the long window. \\
7 & SmoothedVelocity_r_sw_min & Minimum of smoothed GPS velocity within the short window. \\
8 & SmoothedVelocity_r_sw_med & Median of smoothed GPS velocity within the short window.
\end{tabular}

Final version of the algorithm

The first step of the final algorithm aimed to efficiently transform the data from a time series into a classification problem. Bowling events are known to have a high 
acceleration component. PlayerLoadTM is a derived variable from Catapult that represents high acceleration movements (Barrett, Midgley \& Lovell, 2014). Preliminary analysis on training data found that fast bowling delivery instances could be identified by an occurrence of a smoothed PlayerLoadTM of 3.5 or greater. This value can therefore be interpreted as a lowest common denominator of fast bowling delivery events. Every time point where smoothed PlayerLoadTM was 3.5 or greater was identified as a potential delivery and became a candidate event for the random forest section of the algorithm.

High levels of rotation occur during a bowling delivery, and during the iterative process described above, maximal rotation, found by the maximum angular velocity in all three axes, was identified as a key variable. Once candidate events had been identified, these timepoints were realigned to maximal rotation, to closer match the timing of the actual delivery events.

A window of 7 and 5.5 seconds before and after each timepoint was then selected as the length of a typical delivery. This window was chosen following visual inspection of traces to ensure that all the data from the full run up, delivery and follow through were included, but without stretching the time period for too long.

Once the candidate events were identified and realigned through the above process, the second step of the final algorithm consisted of calculating the 8 features selected during the development of the algorithm for each candidate event (Table 1). Predictions were then generated with the random forest model. Receiver Operating Characteristic (ROC) curves were produced to identify the appropriate cut off point for the prediction percentage of the candidate event being an actual delivery.

\section{Validation of the algorithm}

The final iteration of the algorithm was tested using an independent data set including deliveries from fourteen bowlers, different to the original bowlers used during the training and optimisations stages, from 137 sessions (86 matches and 51 training sessions). Video footage and Hawkeye timestamped data was used to manually code when a bowling delivery occurred, and this was compared to the events detected by the algorithm.

\section{Statistical Analysis}

The validity of the algorithm was tested in two conditions: events in matches and events in training. Sensitivity and specificity of the algorithm were calculated by determining the proportion of true or false, positive or negative events detected as defined in Table 2. Matthews Correlation Coefficient (MCC) was used to analyse the relationship between actual events and events detected by the machine learning algorithm on the validation of the final iteration. ROC curves and the Area Under Curve (AUC) were also produced, to analyse the sensitivity and specificity of the algorithm's confidence at predicting bowling deliveries in both conditions.

Table 2. Definitions of true and false, positive and negative bowling deliveries

\begin{tabular}{|c|c|}
\hline True Positive & Delivery bowled, and automatically detected \\
\hline False Positive & Delivery not bowled, but automatically detected \\
\hline True Negative & $\begin{array}{l}\text { Delivery not bowled, an event is detected with a confidence level of less } \\
\text { than } 35 \% \text {, meaning no delivery is automatically detected }\end{array}$ \\
\hline False Negative & Delivery bowled, and not automatically detected \\
\hline
\end{tabular}




\section{RESULTS}

The first iteration of the algorithm detected 782 events, of which 746 were truepositive, 35 were true-negative and 1 was false-negative resulting in a sensitivity of $99.9 \%$ and a specificity of $100.0 \%$ (Table 3 ). The second iteration of the algorithm detected 4174 events, of which 3867 were true-positive, 304 were true-negative, 1 was a false-positive and 2 were false-negative, resulting in a sensitivity of $99.9 \%$ and a specificity of $99.7 \%$ (Table 3). The final iteration of the algorithm detected 12992 events, of which 12247 were true-positive, 707 were true-negative, 11 were falsepositive and 27 were false-negative, resulting in a sensitivity of $99.8 \%$ and a specificity of $98.5 \%$ (Table 3 ).

Table 3. Accuracy of versions of machine learning algorithm to automatically detect bowling deliveries

\begin{tabular}{cccccc}
\hline & Version 1 & Version 2 & Version 3 & Validation - in training & Validation - in matches \\
\hline True Positive & 746 & 3867 & 12247 & 1170 & 2621 \\
False Positive & 0 & 1 & 11 & 6 & 16 \\
True Negative & 35 & 304 & 707 & 339 & 494 \\
False Negative & 1 & 2 & 27 & 45 & $96.6 \%$ \\
Sensitivity & $99.9 \%$ & $99.9 \%$ & $99.8 \%$ & $96.3 \%$ & $96.9 \%$ \\
Specificity & $100.0 \%$ & $99.7 \%$ & $98.5 \%$ & $98.3 \%$ & \\
\hline
\end{tabular}

In the validation of the final algorithm, a high MCC was observed in both conditions between real events and those detected by the algorithm; training ( $r=0.911)$ and matches $(r=0.968)$. In both conditions; training and match, a high level of sensitivity (96.3\% and $99.6 \%$, respectively) and specificity (98.3\% and $96.9 \%$, respectively) were also observed in the validation (Table 3).

The ROC curve for each condition allowed the cut off level for the positive detection of a bowling delivery to be set at 35\% (Figure 1). The AUC (Figure 1) is very similar for both conditions ( 0.987 vs. 0.997 for training vs. matches respectively) indicating the algorithm is equally effective in both training and match conditions. 


\section{ROC Curve}

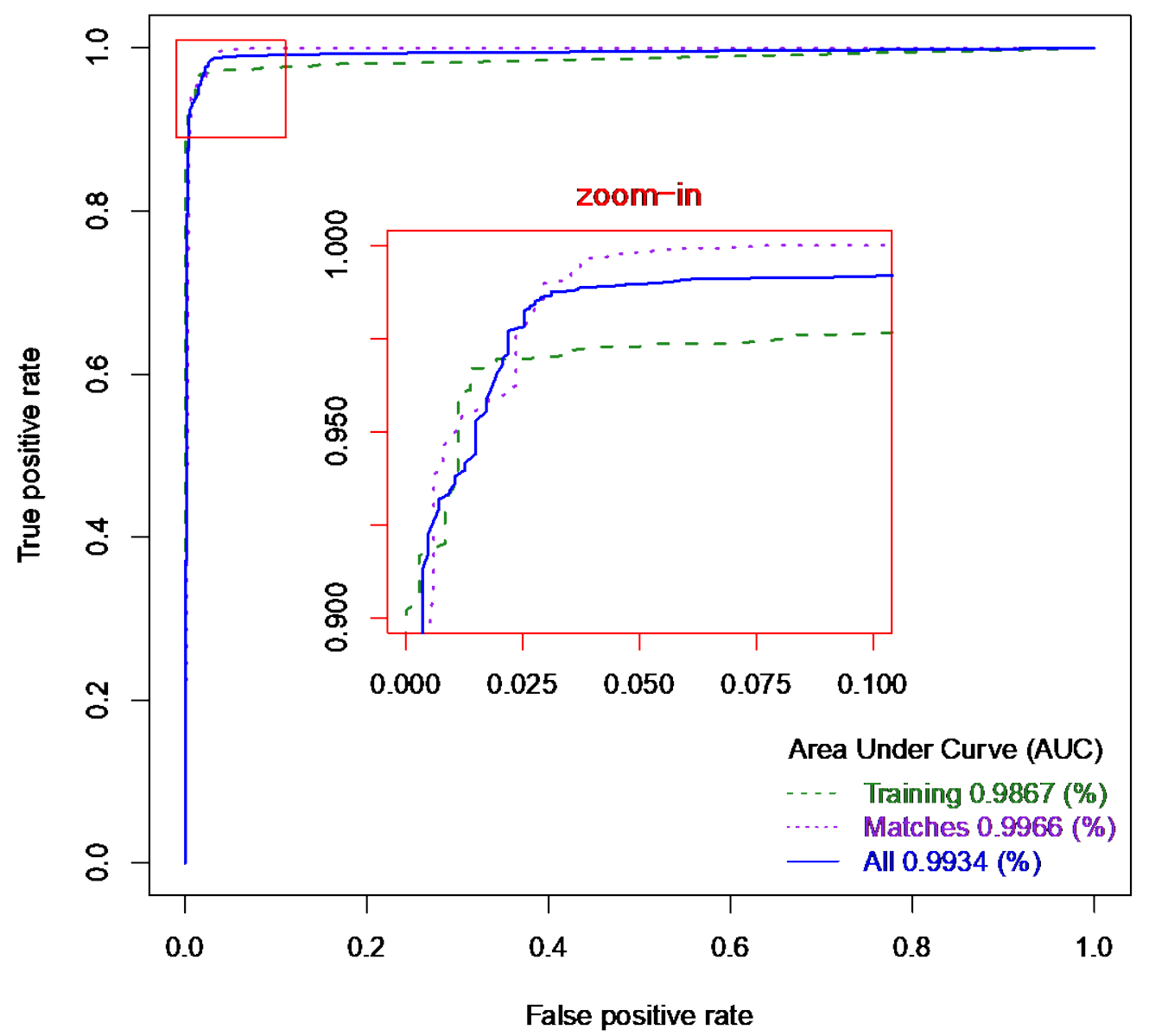

Figure 1. Receiver Operating Characteristic (ROC) curve for training, matches and all deliveries using the validation of the final version of the algorithm.

\section{DISCUSSION}

The purpose of this study was to develop and independently validate a robust machine learning algorithm using GPS, accelerometer and gyroscope data to auto detect cricket bowling deliveries in both training and matches.

The algorithm was shown to be sensitive and specific at detecting bowling deliveries in both training and matches. The sensitivity level observed in training $(96.3 \%)$ was less than that reported by McNamara et al. (2015) (99.0\%) and within the ranges of those reported by McGrath et al. (2019) (93.0\%). The sensitivity levels when using match data (99.6\%), were similar to those reported by McNamara et al. (2015) for matches (99.5\%). Specificity levels were greater than those reported by McNamara et al. (2015) particularly in events occurring in matches (96.9\% vs. $74.0 \%)$. A potential reason for this substantial increase in specificity during matches compared to previous research is that 'real' data was used from both training and matches, rather than manufacturing events such as throwing. Similarly, the higher levels of specificity in training reported by McGrath et al. (2019) of $100.0 \%$, compared to the $98.3 \%$ observed in this study, could be explained by the lack of 'real' data when testing the McGrath et al. (2019) algorithm. It is likely that if it was tested in match conditions, both sensitivity and specificity levels would be reduced as has been observed in other studies recently (McNamara et al. 2015, Murray et al. 2016). Another potential reason for the 
substantial increase in specificity is the large sample size ensuring a large number of false events i.e. throws and dives were analysed alongside true bowling deliveries. After manually looking at a sample of the falsely classified events, it was identified that these were typically low intensity warm up deliveries or a throw in the field preceded by a run.

The data sets used to train and validate the algorithm included a wide range of deliveries at differing intensities including warm up deliveries and those bowled by fast bowlers during rehabilitation to make the data sets as representative as possible of 'real' conditions. The participants were also both male and female and of differing standards (youth, county and international). In the general trade-off between sensitivity and specificity, including a wider range of players favoured sensitivity. It was found that the algorithm was able to detect deliveries bowled at lower intensities, regardless of whether this is youth, female or male bowlers bowling at lower speed in warm-ups, training or matches, while keeping the specificity and overall accuracy to very good levels.

The GPS and inertial sensors used within this study could also be used to provide metrics on performance such as run up speed, deceleration and trunk rotation. These metrics could begin to provide a guide for the intensity of each delivery bowled. This would have several clear benefits; firstly, it allows for an accurate and complete monitoring of bowling workload, that includes deliveries bowled at lower intensities. This has previously been identified to be a potential 'blind spot' and limitation with other workload monitoring techniques (Petersen et al., 2011). Secondly, practitioners and coaches could begin to use these future performance metrics as a gauge as to whether individuals are fully prepared to bowl at match intensity whether coming back from a period of rest or rehabilitation (Hulin et al., 2014; Petersen et al., 2011). Finally, they may also be able to provide coaches with feedback on in game technique which is currently not possible without the use of invasive three-dimensional motion analysis techniques. Further research however is required to determine whether these metrics are correlated with bowling intensity and the performance characteristics of fast bowling (Worthington et al., 2013).

\section{Practical Applications}

Coaches, medical staff and players will be better able to monitor bowling workloads because the detection of bowling deliveries using a Catapult OptimEye S5 unit is not reliant upon an individual observing and manually recording the deliveries.

The algorithm is as sensitive and specific in training as it is in matches, and so a whole picture can be put together when monitoring bowling workloads with minimal deliveries being missed.

These developments will all aid in optimising a fast bowlers training and match schedule to reduce the risk of injury and improve bowling performance as players maintain their optimal state.

\section{CONCLUSIONS}

This study has developed and provided a validation of a machine learning algorithm using large data sets covering many different conditions that successfully predicts fast bowling deliveries. The algorithm is highly sensitive and specific to both match and training deliveries across a wide range of intensities. This tool enables practitioners to monitor bowling workload more accurately and efficiently allowing better tracking and workload management. Future research could investigate the 
relationships between performance metrics derived from the GPS and inertial sensors and bowling intensity and known fast bowling performance characteristics.

\section{REFERENCES}

Barrett, S., Midgley, A., Lovell, R. 2014. PlayerLoad: Reliability, convergent validity, and influence of unit position during treadmill running. International Journal of Sports Physiology and Performance, 9, 945-952.

Breiman, L. 2001. Random forests. Machine Learning, 45, 5-32.

Chicco, D. (2017). Ten quick tips for machine learning in computational biology. Biodata Mining, 10, 1-17.

Dennis, R., Farhart, P., Goumas, C., Orchard, J. 2003. Bowling workload and the risk of injury in elite cricket fast bowlers. Journal of Science and Medicine in Sport, 6, 359-367.

Ferdinands, R., Marshall, R.N., Kersting, U. 2010. Centre of mass kinematics of fast bowling in cricket. Sports Biomechanics, 9, 139-152.

Gastin, P.B., McLean, O., Spittle, M., Breed, R.V.P. 2013. Quantification of tackling demands in professional Australian football using integrated wearable athlete tracking technology. Journal of Science and Medicine in Sport, 16, 589-593.

Genuer, R., Poggi, J-M., Tuleau-Malot, C. 2015. VSURF: A R package for variable selection using random forests. The $\mathrm{R}$ Journal, $\mathrm{R}$ Foundation for Statistical Computing, 7, 19-33.

Hastie, T., Tibshirani, R., Friedman, J. The Elements of Statistical Learning: Data Mining, Inference, and Prediction. New York: Springer; 2009.

Hulin, B.T., Gabbett, T.J., Blanch, P., Chapman, P., Bailey, D., Orchard, J.W. 2014. Spikes in acute workload are associated with increased injury risk in elite cricket fast bowlers. British Journal of Sports Medicine, 48, 708-712.

Hulin, B.T., Gabbett, T.J., Johnston, R.D., Jenkins, D.G. 2017. Wearable microtechnology can accurately identify collision events during professional rugby league match-play. Journal of Science and Medicine in Sport, 20, 638-642.

Jensen, U., Schmidt, M., Hennig, M., Dassler, F.A., Jaitner, T., Eskofier, B.M. 2015. An IMU-based mobile system for golf putting analysis. Sports Engineering, 18, $123-$ 133.

Kelly, D., Coughlan, G.F., Green, B.S., Caulfield, B. 2012. Automatic detection of collisions in elite level rugby union using a wearable sensing device. Sports Engineering, 15, 81-92.

McGrath, J.W., Neville, J., Stewart, T., Cronin, J. 2019. Cricket fast bowling detection in a training setting using an inertial measurement unit and machine learning. Journal of Sports Sciences, 37, 1220-1226.

McNamara, D.J., Gabbett, T.J., Chapman, P., Naughton, G., Farhart, P. 2015. The validity of microsensors to automatically detect bowling events and counts in cricket fast bowlers. International Journal of Sports Physiology and Performance, 10, 7175.

Murray, N.B., Black, G.M., Whiteley, R.J., Gahan, P., Cole, M.H., Utting, A., Gabbett, T.J. 2016. Automatic detection of pitching and throwing events in baseball with inertial measurement sensors. International Journal of Sports Physiology and Performance, 12, 1-18. 
Orchard, J.W., James, T., Portus, M., Kountouris, A., Dennis, R. 2009. Fast bowlers in cricket demonstrate up to 3- to 4-week delay between high workloads and increased risk of injury. American Journal of Sports Medicine, 37, 1186-1192.

Orchard, J.W., Blanch, P., Paoloni, J., Kountouris, A., Sims, K., Orchard, J.J., Brukner, P. 2015. Cricket fast bowling workload patterns as risk factors for tendon, muscle, bone and joint injuries. British Journal of Sports Medicine, 49, 1064-1068.

Petersen, C.J., Pyne, D., Portus, M., Karppinen, S., Dawson B. 2009. Variability in movement patterns during One Day Internationals by a cricket fast bowler. International Journal of Sports Physiology and Performance, 4, 278-281.

Petersen, C., Portus, D., Dawson, M. 2009. Quantifying positional movement patterns in Twenty20 cricket. International Journal of Performance Analysis in Sport, 9, 165170.

Petersen, C.J., Pyne, D., Dawson, B., Portus, M., Kellett, A. 2010. Movement patterns in cricket vary by both position and game format. Journal of Sports Sciences, 28, 45-52.

Petersen, C.J., Pyne, D.B., Dawson, B.T., Kellett, A.D., Portus, M.R. 2011. Comparison of training and game demands of national level cricketers. Journal of Strength and Conditioning Research, 25, 1306-1311.

Pfeiffer, M. Hohmann, A. 2012. Applications of neural networks in training science. Human Movement Science, 31, 344-359.

Ranson, C., Burnett, A., King, M., Patel, N., O'Sullivan, P. 2008. The relationship between bowling action classification and three-dimensional lower trunk motion in fast bowlers in cricket. Journal of Sports Sciences, 26, 267-276.

Ranson, C., Hurley, R., Rugless, L., Mansingh, A., Cole, J. 2013. International cricket injury surveillance: a report of five teams competing in the ICC Cricket World Cup. British Journal of Sports Medicine, 47, 637-643.

Varley, M.C., Fairweather, I.H., Aughey, R.J. 2012. Validity and reliability of GPS for measuring instantaneous velocity during acceleration, deceleration, and constant motion. Journal of Sports Science, 30, 121-127.

Warren, A., William, S., McCaig, S., Trewartha, G. 2018. High acute:chronic workloads are associated with injury in England Wales Cricket Board Development Programme fast bowlers. Journal of Science and Medicine in Sport, 21, 40-45.

Whiteside, D., Cant, O., Connolly, M, Reid, M. 2017. Monitoring hitting load in tennis using inertial sensors and machine learning. International Journal of Sports Physiology and Performance, 12, 1212-1217.

Worthington, P.J., King, M.A., Ranson, C.A. 2013. Relationships between fast bowling technique and ball release speed in cricket. Journal of Applied Biomechanics, 29, 78-84.

Wundersitz, D.W.T., Josman, C., Gupta, R., Netto, K.J., Gastin, P.B., Robertson, S. 2015. Classification of team sport activities using a single wearable tracking device. Journal of Biomechanics, 48, 3975-3981. 